01

\title{
K вопросу о зависимости силы пучково-канального взаимодействия от формы радиальных профилей релятивистского электронного пучка и ионного канала в режиме ионной фокусировки
}

\author{
(C) Е.К. Колесников, А.С. Мануйлов \\ Санкт-Петербургский государственный университет, \\ 198504 Санкт-Петербург, Россия \\ ๑ e-mail: a.manuylov@spbu.ru, man06@mail.ru \\ (Поступило в Редакцию 7 апреля 2016 г.)
}

Получены формулы для расчета силы взаимодействия релятивистского электронного пучка с ионным плазменным каналом в случае транспортировки пучка в режиме ионной фокусировки. Исследовано влияние различия в радиальных профилях пучка и ионного канала на величину указанной силы при разных амплитудах отклонения пучка от оси симметрии канала.

DOI: 10.21883/JTF.2017.02.44145.1845

\section{Введение}

В последние годы внимание исследователей привлекают вопросы транспортировки релятивистских электронных пучков (РЭП) в плотных и разреженных газоплазменных средах [1-11]. Определенное место в данных исследованиях занимает задача нахождения условий устойчивой проводки пучков по предварительно созданным плазменным каналам в режиме ионной фокусировки (ИФ) [5-7].

Известно, что режим ИФ может быть реализован при достаточно низком давлении в фоновой газоплазменной среде, когда электроны плазмы в предварительно созданном плазменном канале при воздействии поперечной составляющей электрического поля фронтальной части РЭП покидают область, занимаемую пучком, не создавая существенной дополнительной ионизации фонового газа [5-7]. В этом случае пучок будет распространяться под действием фокусирующего электрического поля, созданного ионной компонентой плазменного канала, которая в силу достаточно большой массы ионов (по сравнению с массой электронов) определенное время может считаться неподвижной.

В режиме ИФ РЭП может быть подвержен ряду крупномасштабных неустойчивостей, среди которых наибольшим инкрементом нарастания обладает так называемая ионная шланговая неустойчивость (ИШН) [5]. При развитии данной моды пучок испытывает растущие по амплитуде изгибные поперечные колебания в результате электростатического взаимодействия с отстающим по фазе из-за инерционных эффектов ионным каналом. Указанное отставание приводит к раскачке поперечных колебаний как пучка, так и ионного канала.

В настоящей работе исследовано влияние различия в радиальных профилях пучка и ионного канала на величину указанной силы при разных амплитудах отклонения пучка от оси симметрии канала.

\section{Постановка и решение задачи}

Рассмотрим параксиальный моноэнергетический аксиально-симметричный РЭП, распространяющийся в разряженном газе вдоль предварительно созданного плазменного канала.

Выберем цилиндрическую систему координат $(r, \theta, z)$, так, чтобы ось $z$ совпадала с осью симметрии канала. Давление фонового газа полагаем таким, что выполнены условия существования режима ИФ, а именно [5]

$$
b_{a v} \gg R_{b} \text {. }
$$

Здесь $b_{a v}-$ характерная длина развития лавинной ионизации окружающего пучка газа, $R_{b}$ - характерный радиус пучка. В этой ситуации плазменные электроны под действием поперечной компоненты электрического поля в головной части РЭП удаляются из плазменного канала без дополнительной ионизации фонового газа.

Далее будем считать, что радиальные зависимости объемных концентраций электронов пучка и ионов плазменного канала имеют либо гауссов вид, либо беннетовский, а именно

$$
\begin{aligned}
& n_{m}(r)=\frac{N_{m}}{\pi R_{m}^{2}} \exp \left(-\frac{r^{2}}{R_{m}^{2}}\right), \quad m=1,2, \\
& n_{j}(r)=\frac{N_{j}}{\pi R_{j}^{2}} \frac{1}{\left(1+r^{2} / R_{j}^{2}\right)^{2}}, \quad j=1,2,
\end{aligned}
$$

где

$$
N_{1}=N_{b}, N_{2}=N_{i}, R_{1}=R_{b}, R_{2}=R_{i} .
$$

Здесь $N_{b}, N_{i}$ - соответственно погонные концентрации электронов пучка и ионов плазменного канала (концентрации, приходящиеся на единицу длины пучка или ионного канала), $R_{b}, R_{i}$ - характерные радиусы пучка и ионного канала.

В рамках модели „жесткого пучка“ предполагаем, что РЭП смещается в поперечном направлении без деформации его радиального профиля концентрации. В этом 


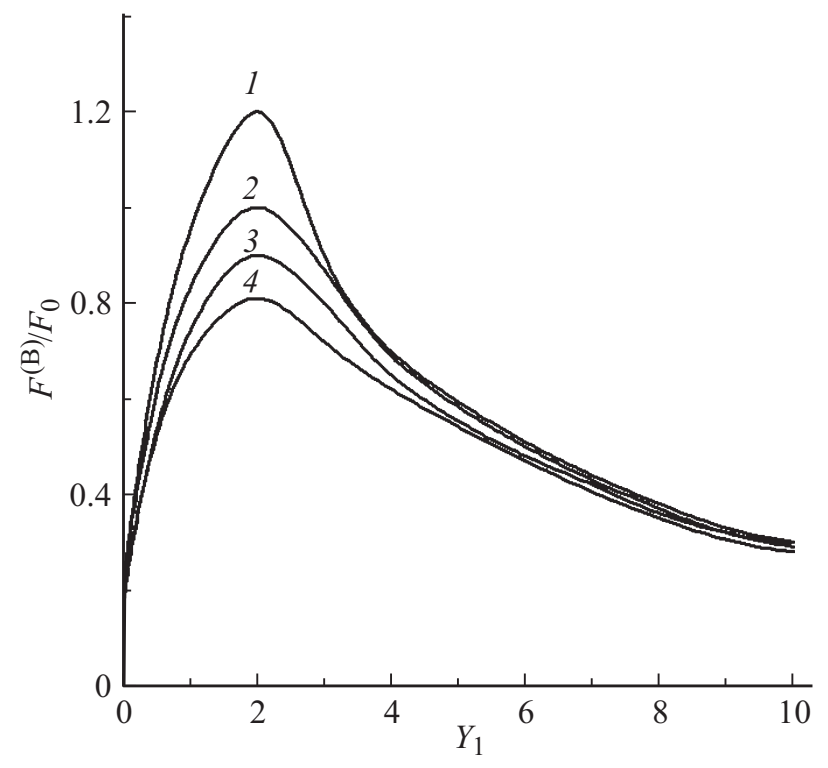

Рис. 1. Зависимости трекинг-силы от приведенной амплитуды отклонения центра симметрии пучка от оси ионного канала при различных значениях параметра $\eta: 1-0.2,2-0.5$, $3-1,4-1.5$.

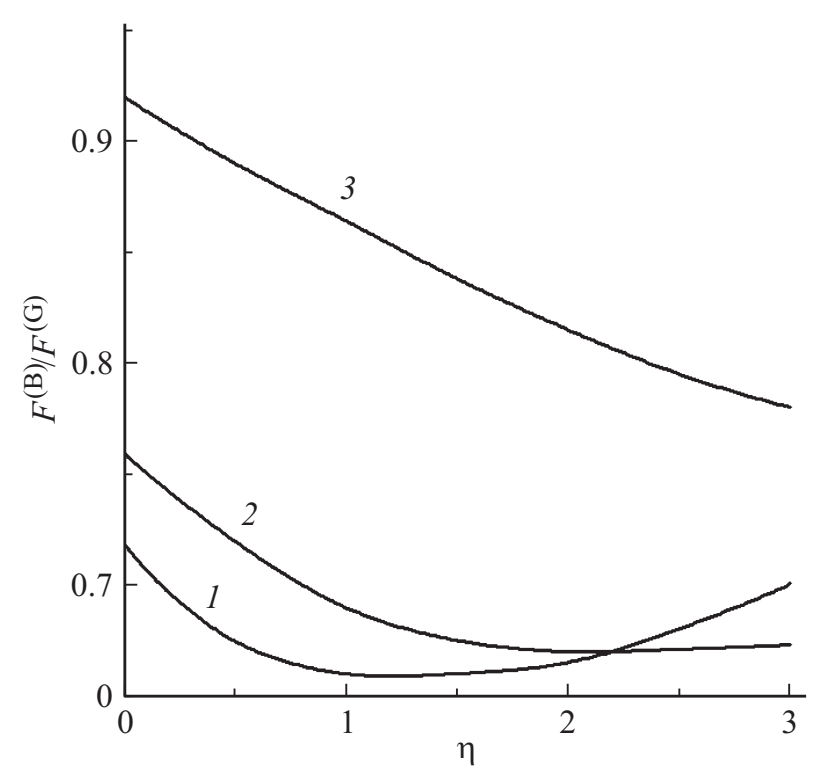

Рис. 2. Зависимость отношения трекинг-сил для беннетовского и гауссовского случаев от параметра $\eta$ при различных значениях приведенной амплитуды отклонения центра симметрии пучка от оси ионного канала $Y_{1}: 1-0.5,2-1,3-4$.

случае трекинг-сила (возвращающая сила), действующая на смещенный пучок со стороны неподвижного ионного канала, может быть определена как

$$
\left|F_{x}\right|=|e| \frac{N_{b}}{\pi R_{b}^{2}} \int_{\Omega} d \mathbf{r}_{\perp} \Phi(r, \theta, S) E_{r}^{(i)} \cos \theta,
$$

где $e-$ заряд электрона, $S^{2}=r^{2}+Y^{2}-2 r Y \cos \theta, \mathbf{r}_{\perp}-$ радиус-вектор, проведенный из точки пересечения поперечной плоскости и оси ионного канала в точку наблюдения, $\Omega$ - область поперечной плоскости, занятая пучково-плазменной системой, $\theta-$ угол между осью $x$, вдоль которой происходит смещение пучка, и направлением радиус-вектора $\mathbf{r}_{\perp}, Y-$ амплитуда отклонения центра симметрии пучка относительно оси ионного канала, функция $\Phi(r, \theta, S)$ в случае гауссовых и беннетоских радиальных профилей пучка и ионного канала имеет следующий вид соответственно

$$
\Phi(r, \theta, S)=\exp \left(-\frac{S^{2}}{R_{b}^{2}}\right), \quad \Phi(r, \theta, S)=\frac{1}{\left(1+S^{2}\right)^{2}},
$$

где $E_{r}^{(i)}$ - радиальная компонента напряженности электростатического поля, созданного ионным каналом в точке наблюдения.

В силу предположения о неподвижности ионной компоненты канала имеем

$$
\frac{1}{r} \frac{d}{d r}\left(r E_{r}^{(i)}\right)=4 \pi|e| n_{i}(r)
$$

с граничным условием $\left.E_{r}^{(i)}\right|_{r=0}=0$.

Тогда в случае гауссового радиального профиля ионного канала можно получить

$$
E_{r}^{(i)}(r)=\frac{2 \alpha_{n} I_{b}}{r \beta c}\left[1-\exp \left(-\frac{r^{2}}{R_{i}^{2}}\right)\right],
$$

где $\alpha_{n}=N_{i} / N_{b}$ - коэффициент зарядовой нейтрализации в режиме ИФ, $I_{b}-$ полный ток пучка, $c$ скорость света, $\beta=v_{z} / c-$ отношение продольной компоненты скорости частиц пучка (который полагается моноскоростным в $z$-направлении) к скорости света.

Аналогичным образом в случае беннетовского радиального профиля ионного канала нетрудно получить

$$
E_{r}^{(i)}(r)=\frac{2 \alpha_{n} I_{b}}{\beta c} \frac{r / R_{i}^{2}}{\left(1+r^{2} / R_{i}^{2}\right)} .
$$

Для упрощения вычислений введем следующие безразмерные величины:

$$
r_{1}=\frac{r}{R_{b}}, \quad Y_{1}=\frac{Y}{R_{b}}, \quad \eta=\left(\frac{R_{i}}{R_{b}}\right)^{2} .
$$

Тогда с учетом (6), (7), (9) и (10) имеем соответственно для гауссова и беннетовского случаев:

$$
\begin{aligned}
F^{(G)}= & F_{0} \int_{0}^{2 \pi} d \theta \cos \theta \int_{0}^{\infty} d r_{1} \exp \left[-\left(r_{i}^{2}+Y_{1}^{2}-2 r_{1} Y_{1} \cos \theta\right)\right] \\
& \times\left[1-\exp \left(-r_{1}^{2} / \eta\right)\right], \\
F^{(B)} & =F_{0} \int_{0}^{2 \pi} d \theta \cos \theta \int_{0}^{\infty} d r_{1} \frac{r_{1}}{\left(1+r_{1}^{2}+Y_{1}^{2}-2 r_{1} Y_{1} \cos \theta\right)^{2}} \\
& \times \frac{r_{1} / \eta}{\left(1+r_{1}^{2} / \eta\right)} .
\end{aligned}
$$

где $F_{0}=2 f_{n} I_{b}^{2} /\left[\pi R_{b}(\beta c)^{2}\right]-$ характерная трекинг-сила, приходящаяся на единицу длины пучка. 
На рис. 1 представлены графики зависимости трекингсилы $F^{(B)}$ от $Y_{1}$, определенной по формуле $(12)$ и нормированной к характерной силе $F_{0}$. Кривые построены при разных значениях параметра $\eta$. Кривая 1 соответствует случаю $\eta=0.2,2-\eta=0.5,3-\eta=1,4-\eta=1.5$. Из рисунка следует, что с ростом параметра $\eta$ трекингсила $F^{(B)} / F_{0}$ убывает. Это объясняется тем, что при увеличении параметра $\eta$ ионный канал становится шире пучка. При этом часть ионного канала, находящегося „вне“ пучка, работает на удаление РЭП от оси системы. Поэтому трекинг-сила убывает. Кроме того, из рис. 1 видно, что указанная сила имеет явно выраженный максимум.

На рис. 2 представлена зависимость $F^{(B)} / F^{(G)}$ от параметра $\eta$ при разных значениях амплитуды $Y_{1}$. Кривая 1 соответствует $Y_{1}=0.5,2-Y_{1}=1,3-Y_{1}=4$. Из данного рисунка следует, что $F^{(B)}$ меньше $F^{(G)}$ при любых значениях параметра $\eta$ и амплитуды отклонения пучка $Y_{1}$. При этом при малых значениях $Y_{1}<1$ и значениях параметра $0<\eta \leq 2.5$ величина $F^{(B)} / F^{(G)} \sim 0.7-0.75$.

\section{Заключение}

Рассмотрена задача расчета силы взаимодействия ионного канала и релятивистского электронного пучка, испытывающего поперечное отклонение в случае распространения пучка в режиме ионной фокусировки. Найдены формулы для расчета указанной силы в случае беннетовских и гауссовых радиальных профилей ионного канала и плотности тока пучка. Показано, что рассматриваемая сила существенно зависит от амплитуды отклонения пучка, вида радиальных профилей канала и пучка, а также соотношения их характерных радиальных масштабов.

\section{Список литературы}

[1] Рухадзе А.А., Богданкевич Л.С., Росинский С.Е., Рухлин В.Г. Физика сильноточных релятивистских электронных пучков. М.: Атомиздат, 1980. 167 с.

[2] Миллер P. Введение в физику сильноточных пучков заряженных частиц. М.: Мир, 1984. 432 с.

[3] Колесников Е.К., Мануйлов А.С., Филиппов Б.В. Динамика пучков заряженных частиц в газоплазменных средах. СПб.: Изд-во СПбГУ, 2002. 98 с.

[4] Колесников Е.К., Мануйлов А.С., Зеленский А.Г. Динамика релятивистских электронных пучков в режиме ионной фокусировки. Воскресенск: Изд-во Позитив, 2013. 104 с.

[5] Buchanan H.L. // Phys. Fluids. 1987. Vol. 30. N 1. P. 221-231.

[6] Swanekamp S.B., Hollowey J.P., Kammash T., Gilgenbach R.M. // Phys. Fluid. B. 1992. Vol. 4. N 5. P. $1332-1348$.

[7] Fernsler R.F., Hubbard R.F., Slinker S.P. // Phys. Fluid. B. 1992. Vol. 4. N 12. P. 4153-4165.

[8] Mamedov B.A. // Astropart. Phys. 2015. Vol. 64. P. 1-3.

[9] Колесников Е.К., Мануйлов А.С. // РиЭ. 1992. Т. 37. № 4. C. 694-699.

[10] Колесников Е.К., Мануйлов А.С. // ЖТФ. 2000. Т. 70. Вып. 5. С. 68-73.

[11] Зеленский А.Г., Колесников Е.К. // ЖТФ. 2005. Т. 75. Вып. 11. С. 127-129. 